\title{
Gaussian and Mean curvature of subdivision surfaces*
}

\author{
Jörg Peters**, Georg Umlauf**
}

March 9, 2000

\begin{abstract}
By explicitly deriving the curvature of subdivision surfaces in the extraordinary points, we give an alternative, more direct account of the criteria necessary and sufficient for achieving curvature continuity than earlier approaches that locally parametrize the surface by eigenfunctions.

The approach allows us to rederive and thus survey the important lower bound results on piecewise polynomial subdivision surfaces by Prautzsch, Reif, Sabin and Zorin, as well as explain the beauty of curvature continuous constructions like Prautzsch's. The parametrization neutral perspective gives also additional insights into the inherent constraints and stiffness of subdivision surfaces.
\end{abstract}

\section{Introduction}

Almost all subdivision algorithms in the current literature achieve tangent continuity but not curvature continuity. We give a simple characterization of the causes underlying this phenomenon by explicitly expressing Gaussian and mean curvature in the minimally smooth extraordinary points. This allows us to rederive and thereby survey the important lower bound results of [Sabin '91, Reif '96, Zorin '98, Prautzsch \& Reif '99] and constructions for curvature continuous piecewise polynomial subdivision algorithms by [Prautzsch '97, Prautzsch \& Umlauf '98b, Reif '98b]. Beyond this we get additional insights into the inherent constraints and stiffness of such subdivision algorithms. Since a subdivision surface consists of an infinite collection of polynomial pieces around every extraordinary point one might expect such surfaces to be more flexible than spline surfaces. However, we will see that the infinite application of the same subdivision rule enforces strict rules on the piecewise polynomial rings converging towards extraordinary points. For example, the Jacobian of the subdominant eigenfunctions of a curvature continuous subdivision algorithm must have lower degree than the Jacobian of the subdivision surface itself.

The paper is organized as follows. With the notation of Section 2, we express in each subdivision step $m$ the curvatures of the innermost spline ring around a given extraordinary point as $K_{m}=\left(\mu / \lambda^{2}\right)^{2 m} f_{K}^{m}(u, v)$, respectively, $H_{m}=\left(\mu / \lambda^{2}\right)^{m} f_{H}^{m}(u, v)$ for scalar constants $\mu<\lambda$ and rational functions $f$. The factor $\mu / \lambda^{2}$ immediately implies necessary constraints on curvature continuous subdivision surfaces and the weakest form of curvature smoothness: the principal curvatures of piecewise polynomial $C^{1}$ subdivision algorithms are square integrable. Section 4 derives and reviews necessary constraints on subdivision surfaces to be curvature continuous by observing that $f$ should be constant in the limit and equating the degree of numerator and denominator of $f$ at the extraordinary point. Section 5 reviews Prautzsch's sufficient condition and his unique construction of a curvature continuous, linear, stationary subdivision algorithm by projection.

\footnotetext{
* Supported by NSF grant CCR-9901894.

${ }^{* *}$ CISE, University of Florida, P.O. Box 116120, Gainesville, FL 32611-6120, USA, e-mail: [jorg|umlauf]@cise.ufl.edu.
} 


\section{Notation and basic facts}

In this section we define just the basic notation and facts needed for our analysis; for a formal, more general and abstract setting, the reader is referred to [Reif '98a] and [Zorin '98].

While our analysis applies to a larger class of subdivision algorithms, we focus in the following on generalized box-spline subdivision algorithms, that is on affine invariant, symmetric, linear, local, stationary algorithms that generalize box-spline subdivision and generate (regular) $C^{1}$ surfaces. In particular, the limit surface has a piecewise polynomial parametrization and the parametric smoothness between the pieces is well-known except at a finite number of extraordinary points. An extraordinary point is the limit point of a minimal subnet of the initial control net under repeated application of the subdivision algorithm. Such a subnet consists of an $n$-valent vertex (for a primal subdivision algorithm) or an $n$-sided facet (for a dual subdivision algorithm) and just those neighboring control points that determine a surface ring $\mathbf{x}_{0}$ around an $n$-sided hole by the regular box-spline subdivision rules (Figure 1 left).
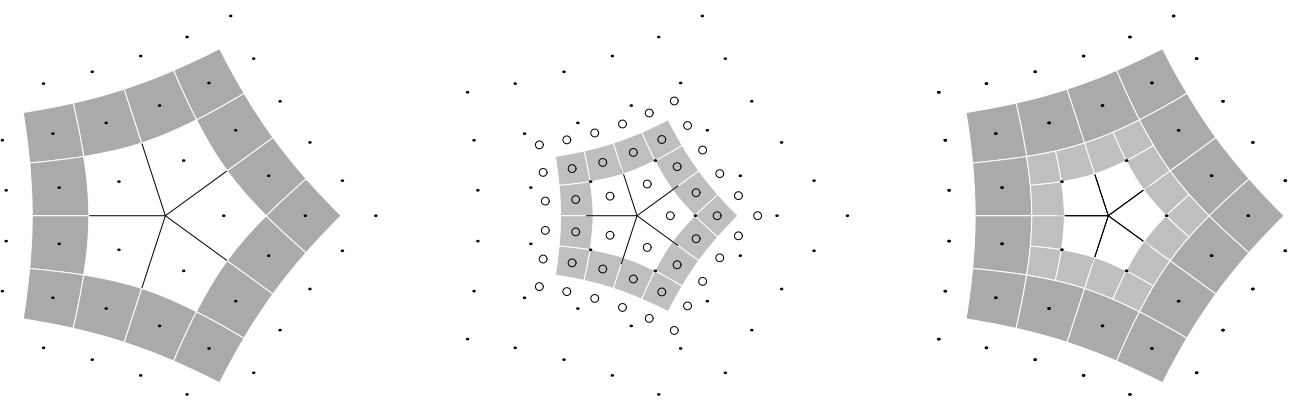

Figure 1: Inserting a surface ring $\mathbf{x}_{m}$ (light grey) into the hole left by $\mathbf{x}_{m-1}$ (dark grey). $\mathbf{x}_{m}$ consists of 5 images of $2 \square \backslash \square$. The vector of control points $\mathbf{C}_{m}$ of $\mathbf{x}_{m}$ (circles) is obtained by one subdivision step applied to $\mathbf{C}_{m-1}$ (dots).

If we arrange the points of the subnet into the column vector $\mathbf{C}_{0}$ then each subdivision step transforms the subnet by applying the same square, stochastic subdivision matrix $A$. After $m$ applications the result is the subnet

$$
\mathbf{C}_{m}=A^{m} \mathbf{C}_{0}
$$

The $m$ th subdivision step adds the surface ring $\mathbf{x}_{m}(u, v)$ inside the hole left by the $(m-1)$ st subdivision step (Figure 1 right). The surface rings are box-splines and can therefore be represented as

$$
\mathbf{x}_{m}:\{0, \ldots, n-1\} \times \Omega \rightarrow \mathbb{R}^{3}, \quad \mathbf{x}_{m}(u, v)=\mathbf{B}(u, v) \mathbf{C}_{m},
$$

where $\mathbf{B}(u, v)$ is the row vector of the box-spline basis functions and the domain $\Omega$ is either $2 \square \backslash \square$ or $2 \triangle \backslash \triangle$, with $\square$ the unit square and $\triangle$ the unit triangle.

For simplicity we assume that $A$ is diagonalizable with eigenvalues

$$
1=\lambda_{0}>\underbrace{\lambda_{1}=\lambda_{2}}_{=: \lambda}>\underbrace{\lambda_{3}=\lambda_{4}=\lambda_{5}}_{=: \mu}>\cdots \geq 0,
$$

such that $\lambda_{1}=\lambda_{2}$ correspond to the 1st and $(n-1)$ st block, $\lambda_{3}=\lambda_{4}$ (for $n>3$ ) to the 2nd and $(n-2)$ nd block and $\lambda_{5}$ to the 0 th block of the Fourier decomposition of $A$ (c.f. [Peters \& Reif '98]). The corresponding eigenvectors are denoted by $\mathbf{v}_{i}$, i.e. $A \mathbf{v}_{i}=\lambda_{i} \mathbf{v}_{i}$ for all $i$. For subdivision algorithms with more general subdivision matrices $A$ the reader is referred to [Reif '98a, Zorin '98]. 
In terms of multiples $\mathbf{p}_{i}$ of the eigenvectors the subnet can be expressed as

$$
\mathbf{C}_{m}=\sum_{i} \lambda_{i}^{m} \mathbf{v}_{i} \mathbf{p}_{i}, \quad \mathbf{p}_{i} \in \mathbb{R}^{3}
$$

Expanded in the eigenfunction $\mathbf{e}^{i}:\{0, \ldots, n-1\} \times \Omega \rightarrow \mathbb{R},(u, v) \mapsto \mathbf{B}(u, v) \mathbf{v}_{i}$ associated with $\mathbf{v}_{i}$ the surface ring $\mathbf{x}_{m}$ is of the form

$$
\mathbf{x}_{m}(u, v)=\sum_{i} \lambda_{i}^{m} \mathbf{B}(u, v) \mathbf{v}_{i} \mathbf{p}_{i}=\sum_{i} \lambda_{i}^{m} \mathbf{e}^{i}(u, v) \mathbf{p}_{i}
$$

A well-known fact of differential geometry (see e.g. [Carmo '76]) is that for any regular surface parametrization $\mathbf{x}$ the Gauss curvature $K$ and the mean curvature $H$ are

$$
\begin{aligned}
K(u, v) & =\frac{e(u, v) g(u, v)-f(u, v)^{2}}{E(u, v) G(u, v)-F(u, v)^{2}} \\
H(u, v) & =\frac{e(u, v) G(u, v)-2 f(u, v) F(u, v)+g(u, v) E(u, v)}{2\left(E(u, v) G(u, v)-F(u, v)^{2}\right)}
\end{aligned}
$$

where $\mathbf{x}_{u}$ is the partial derivative of $\mathbf{x}(u, v)$ with respect to $u$

$$
\begin{aligned}
E & =\mathbf{x}_{u} \mathbf{x}_{u}^{t}, \quad F=\mathbf{x}_{u} \mathbf{x}_{v}^{t}, \quad G=\mathbf{x}_{v} \mathbf{x}_{v}^{t}, \\
e=\mathbf{n x}_{u u}^{t}, & f=\mathbf{n} \mathbf{x}_{u v}^{t}, \quad g=\mathbf{n} \mathbf{x}_{v v}^{t},
\end{aligned}
$$

and $\mathbf{n}=\left(\mathbf{x}_{u} \times \mathbf{x}_{v}\right) /\left\|\mathbf{x}_{u} \times \mathbf{x}_{v}\right\|$ is the normal. Since $\mathbf{x}$ is assumed to be regular, the denominators of (1), $E G-F^{2}=\left\|\mathbf{x}_{u} \times \mathbf{x}_{v}\right\|^{2}$, are nonzero and we have

$$
\begin{aligned}
K & =\frac{\operatorname{det}\left(\mathbf{x}_{u}, \mathbf{x}_{v}, \mathbf{x}_{u u}\right) \operatorname{det}\left(\mathbf{x}_{u}, \mathbf{x}_{v}, \mathbf{x}_{v v}\right)-\operatorname{det}\left(\mathbf{x}_{u}, \mathbf{x}_{v}, \mathbf{x}_{u v}\right)^{2}}{\left\|\mathbf{x}_{u} \times \mathbf{x}_{v}\right\|^{4}} \\
H & =\frac{\operatorname{det}\left(\mathbf{x}_{u}, \mathbf{x}_{v}, \mathbf{x}_{u u}\right)\left(\mathbf{x}_{v} \mathbf{x}_{v}^{t}\right)-2 \operatorname{det}\left(\mathbf{x}_{u}, \mathbf{x}_{v}, \mathbf{x}_{u v}\right)\left(\mathbf{x}_{u} \mathbf{x}_{v}^{t}\right)+\operatorname{det}\left(\mathbf{x}_{u}, \mathbf{x}_{v}, \mathbf{x}_{v v}\right)\left(\mathbf{x}_{u} \mathbf{x}_{u}^{t}\right)}{2\left\|\mathbf{x}_{u} \times \mathbf{x}_{v}\right\|^{3}} .
\end{aligned}
$$

\section{Gauss curvature and mean curvature}

In this section, we derive the Gauss curvature and the mean curvature of the limit surfaces of generalized box-spline subdivision algorithms at extraordinary points. We expand each of the surface rings $\mathbf{x}_{m}$ in terms of the eigenfunctions $\mathbf{e}^{i}$. This approach goes back at least to [Reif '93]. However, in contrast to [Reif '93] we do not analyze the curvature by parametrizing the limit surface locally as a function over the subdominant eigenfunctions $\mathbf{e}^{1}$ and $\mathbf{e}^{2}$ but rather compute the curvature expansion explicitly. That is, we determine the curvatures $K_{m}(u, v)$ and $H_{m}(u, v)$ of $\mathbf{x}_{m}(u, v)$ and then take the limit as $m \rightarrow \infty$.

For simplicity we write $\mathbf{x}$ instead of $\mathbf{x}_{m}$ in the following. Since the basis functions form a partition of unity, $\mathbf{e}^{0} \equiv 1$ and

$$
\begin{aligned}
\mathbf{x}_{u} & =\lambda^{m}\left(\mathbf{e}_{u}^{1} \mathbf{p}_{1}+\mathbf{e}_{u}^{2} \mathbf{p}_{2}\right)+\mu^{m}\left(\mathbf{e}_{u}^{3} \mathbf{p}_{3}+\mathbf{e}_{u}^{4} \mathbf{p}_{4}+\mathbf{e}_{u}^{5} \mathbf{p}_{5}\right)+o\left(\mu^{m}\right), \\
\mathbf{x}_{u v} & =\lambda^{m}\left(\mathbf{e}_{u v}^{1} \mathbf{p}_{1}+\mathbf{e}_{u v}^{2} \mathbf{p}_{2}\right)+\mu^{m}\left(\mathbf{e}_{u v}^{3} \mathbf{p}_{3}+\mathbf{e}_{u v}^{4} \mathbf{p}_{4}+\mathbf{e}_{u v}^{5} \mathbf{p}_{5}\right)+o\left(\mu^{m}\right) .
\end{aligned}
$$

Symmetry in $u$ and $v$ yields the analogous terms for $\mathbf{x}_{v}, \mathbf{x}_{u u}$ and $\mathbf{x}_{v v}$. With the abbreviations

$$
\begin{aligned}
\Delta_{i j} & :=\mathbf{e}_{u}^{i} \mathbf{e}_{v}^{j}-\mathbf{e}_{u}^{j} \mathbf{e}_{v}^{i}, \\
D_{s t}^{i} & :=\Delta_{12} \mathbf{e}_{s t}^{i}-\Delta_{1 i} \mathbf{e}_{s t}^{2}+\Delta_{2 i} \mathbf{e}_{s t}^{1}, \quad s, t \in\{u, v\}, \\
P_{i j} & :=\operatorname{det}\left(\mathbf{p}_{1}, \mathbf{p}_{2}, \mathbf{p}_{i}\right) \operatorname{det}\left(\mathbf{p}_{1}, \mathbf{p}_{2}, \mathbf{p}_{j}\right),
\end{aligned}
$$


it is now easy to see that

$$
\begin{aligned}
\mathbf{x}_{u} \times \mathbf{x}_{v} & =\lambda^{2 m} \Delta_{12}\left(\mathbf{p}_{1} \times \mathbf{p}_{2}\right)+o\left(\lambda^{2 m}\right) \\
\operatorname{det}\left(\mathbf{x}_{u}, \mathbf{x}_{v}, \mathbf{x}_{u u}\right) & =\lambda^{2 m} \mu^{m} \sum_{i=3,4,5} \operatorname{det}\left(\mathbf{p}_{1}, \mathbf{p}_{2}, \mathbf{p}_{i}\right) D_{u u}^{i}+o\left(\lambda^{2 m} \mu^{m}\right) .
\end{aligned}
$$

Symmetry yields the analogous terms for $\operatorname{det}\left(\mathbf{x}_{u}, \mathbf{x}_{v}, \mathbf{x}_{u v}\right)$ and $\operatorname{det}\left(\mathbf{x}_{u}, \mathbf{x}_{v}, \mathbf{x}_{v v}\right)$ and

$$
K_{m}=\left(\frac{\mu}{\lambda^{2}}\right)^{2 m} \frac{\sum_{i, j=3,4,5} P_{i j}\left(D_{u u}^{i} D_{v v}^{j}-D_{u v}^{i} D_{u v}^{j}\right)+o(1)}{\Delta_{12}^{4}\left\|\mathbf{p}_{1} \times \mathbf{p}_{2}\right\|^{4}+o(1)} .
$$

All dependencies on $m$ in the equality are either explicit or hidden in the $o(1)$ terms. We note that $\Delta_{12}$ is the Jacobi determinant of the characteristic map [Reif '93, Reif '95], which is non-zero if the characteristic map is regular, and that $\left\|\mathbf{p}_{1} \times \mathbf{p}_{2}\right\|$ is positive for almost all initial control nets $\mathbf{C}_{0}$. The leading factor of the expression (2) for the Gauss curvature readily yields the following basic characterization of the curvature at extraordinary points (c.f. [Reif' 93 , page 25])

Observation 1 Let $A$ be the subdivision matrix of a regular $C^{1}$ generalized box-spline subdivision algorithm as defined in Section 2 with eigenvalues $1>\lambda>\mu>\ldots \geq 0$.

(a) If $\mu>\lambda^{2}$ then the Gauss curvature at the extraordinary point is infinite.

(b) If $\mu<\lambda^{2}$ then the Gauss curvature at the extraordinary point is zero.

(c) If $\mu=\lambda^{2}$ then the Gauss curvature at the extraordinary point is bounded by the second factor of (2), but is possibly non-unique.

Examples for (a) are [Catmull \& Clark'78, Loop '87, Qu '90], for (b) are [Prautzsch \& Umlauf '98a, Prautzsch \& Umlauf '98b] and for (c) are [Sabin '91, Holt '96]. Note the curious combination of tangent continuity and infinite curvature for the standard algorithms in (a). In case (c), the limit for $m \rightarrow \infty$ yields at the extraordinary point

$$
K=\sum_{i, j=3,4,5} \frac{P_{i j}}{\left\|\mathbf{p}_{1} \times \mathbf{p}_{2}\right\|^{4}} \frac{D_{u u}^{i} D_{v v}^{j}-D_{u v}^{i} D_{u v}^{j}}{\Delta_{12}^{4}} .
$$

Recall that the factor $\left(D_{u u}^{i} D_{v v}^{j}-D_{u v}^{i} D_{u v}^{j}\right) / \Delta_{12}^{4}$ is a rational function in $u$ and $v$. In order for the Gauss curvature to be well-defined at the extraordinary point rather than multi-valued or divergent, $K$ must be constant. Since the $P_{i j}$ depend on the initial net, they can be arbitrary except for $P_{i j}=P_{j i}$ and each summand has to be constant. We conclude that the eigenfunctions $\mathbf{e}^{1}, \ldots, \mathbf{e}^{5}$ must satisfy the six partial differential equations:

$$
\begin{aligned}
D_{u u}^{i} D_{v v}^{j}-2 D_{u v}^{i} D_{u v}^{j}+D_{v v}^{i} D_{u u}^{j} & =\Delta_{12}^{4} \cdot \text { const }_{i j}, & & \text { for } i, j \in\{3,4,5\}, j>i \\
D_{u u}^{i} D_{v v}^{i}-\left(D_{u v}^{i}\right)^{2} & =\Delta_{12}^{4} \cdot \text { const }_{i i}, & & \text { for } i=3,4,5 .
\end{aligned}
$$

Lemma 2 The limit surface of a generalized box-spline subdivision algorithm with $\Delta_{12} \neq 0$ has continuous, for almost all initial nets non-zero, Gauss curvature at the extraordinary point if and only if $\mu=\lambda^{2}$ and the differential equations (4) hold.

Similarly, with $\tilde{P}_{i k l}:=\operatorname{det}\left(\mathbf{p}_{1}, \mathbf{p}_{2}, \mathbf{p}_{i}\right)\left(\mathbf{p}_{k} \mathbf{p}_{l}^{t}\right)$ and $\varepsilon_{k l}=1 / 2$ for $k=l$ and 1 otherwise, the mean curvature is

$$
H_{m}=\left(\frac{\mu}{\lambda^{2}}\right)^{m} \frac{\sum_{\substack{i=3,4,5 \\ k, l=1,2, k \geq l}} \tilde{P}_{i k l}\left(\varepsilon_{k l} D_{u u}^{i}\left(\mathbf{e}_{v}^{k} \mathbf{e}_{v}^{l}\right)-D_{u v}^{i}\left(\mathbf{e}_{u}^{k} \mathbf{e}_{v}^{l}+\mathbf{e}_{v}^{k} \mathbf{e}_{u}^{l}\right)+\varepsilon_{k l} D_{v v}^{i}\left(\mathbf{e}_{u}^{k} \mathbf{e}_{u}^{l}\right)\right)+o(1)}{\Delta_{12}^{3}\left\|\mathbf{p}_{1} \times \mathbf{p}_{2}\right\|^{3}+o(1)} .
$$


The expression for $H_{m}$ yields Observation 1, with Gauss curvature replaced by mean curvature. For $\mu=\lambda^{2}$ we get bounded, not forcibly zero, but possibly non-unique mean curvature

$$
H=\sum_{\substack{i=3,4,5 \\ k, l=1,2, k \geq l}} \frac{\tilde{P}_{i k l}}{\left\|\mathbf{p}_{1} \times \mathbf{p}_{2}\right\|^{3}} \frac{\varepsilon_{k l} D_{u u}^{i}\left(\mathbf{e}_{v}^{k} \mathbf{e}_{v}^{l}\right)-D_{u v}^{i}\left(\mathbf{e}_{u}^{k} \mathbf{e}_{v}^{l}+\mathbf{e}_{v}^{k} \mathbf{e}_{u}^{l}\right)+\varepsilon_{k l} D_{v v}^{i}\left(\mathbf{e}_{u}^{k} \mathbf{e}_{u}^{l}\right)}{\Delta_{12}^{3}} .
$$

In analogy to Lemma 2 the necessary and sufficient conditions for a mean-curvature continuous limit surface require that nine partial differential equations hold:

$$
\varepsilon_{k l} D_{u u}^{i}\left(\mathbf{e}_{v}^{k} \mathbf{e}_{v}^{l}\right)-D_{u v}^{i}\left(\mathbf{e}_{u}^{k} \mathbf{e}_{v}^{l}+\mathbf{e}_{v}^{k} \mathbf{e}_{u}^{l}\right)+\varepsilon_{k l} D_{v v}^{i}\left(\mathbf{e}_{u}^{k} \mathbf{e}_{u}^{l}\right)=\Delta_{12}^{3} \cdot \text { const }_{i k l},
$$

for $i=3,4,5, k, l=1,2, k>l$.

Since the principal curvatures at a point on the $m$ th surface ring are

$$
\kappa_{1,2}^{m}=H_{m} \pm \sqrt{H_{m}^{2}-K_{m}},
$$

(2) and (5) imply that $\kappa_{1}^{m}$ and $\kappa_{2}^{m}$ converge like $O\left(\mu^{m} / \lambda^{2 m}\right)$ for $m \rightarrow \infty$.

Observation 3 The limit surface of a generalized box-spline subdivision algorithm with $\Delta_{12} \neq 0$ is curvature continuous at the extraordinary point if $\mu=\lambda^{2}$ and the differential equations (4) and (7) hold.

Since $\int d \mathbf{x}_{m}=O\left(\lambda^{2 m}\right)$ and $\mu<\lambda$

$$
\sum_{m} \int_{\mathbf{x}_{m}}\left|\kappa_{1,2}^{m}\right|^{2} d \mathbf{x}_{m}=\sum_{m} O\left(\mu^{2 m} / \lambda^{2 m}\right)<\infty .
$$

This immediately implies an interesting fact derived for general $L^{p}$ spaces in [Reif \& Schröder '00].

Observation 4 The principal curvatures of the limit surface of a generalized box-spline subdivision algorithm are square integrable.

\section{Lower bounds on the degree}

We now take a look at the important lower bound results of [Sabin '91, Reif '96, Zorin '98, Prautzsch \& Reif '99]. For this it is crucial to distinguish between the apparent or formal degree of box-spline eigenfunctions, possibly the result of degree-raising, and the true degree denoted by "deg". The true degree is defined to be the minimal number of non-vanishing derivatives. We focus on the differential equations resulting from the Gaussian curvature - the analysis of the mean curvature yields the same results.

We recall that the left hand side of the differential equations (4) are for $i, j=3,4,5$,

$$
\begin{aligned}
G_{i j} & :=D_{u u}^{i} D_{v v}^{j}-2 D_{u v}^{i} D_{u v}^{j}+D_{v v}^{i} D_{u u}^{j}, \quad j>i \\
G_{i i} & :=D_{u u}^{i} D_{v v}^{i}-\left(D_{u v}^{i}\right)^{2} .
\end{aligned}
$$

Let $d=\operatorname{deg}\left(\mathbf{x}_{0}\right)$ denote the total degree, respectively, the bi-degree of a regular box-spline parametrization. A straightforward count yields for all $i, j=3,4,5, j \geq i$, that

- for the total degree $\operatorname{deg}\left(G_{i j}\right) \leq 2(2(d-1)+d-2)=6 d-8$ and

- for the bi-degree $\operatorname{deg}\left(G_{i j}\right) \leq 2(2 d-1+d-1)=6 d-4$,

whereas for the right hand side of (4) 
- the formal total degree of $\Delta_{12}^{4}$ is $4(2 d-2)$ and

- the formal bi-degree of $\Delta_{12}^{4}$ is $4(2 d-1)$.

This degree mismatch implies the following observation.

Observation 5 The limit surface of a generalized box-spline subdivision algorithm with total degree

$$
\operatorname{deg}\left(\mathbf{x}_{0}\right)=d \quad \text { and } \quad \operatorname{deg}\left(\Delta_{12}\right)=2(d-1)
$$

is curvature continuous at an extraordinary point $P$ if and only if $P$ is a flat point, i.e. $\mu<\lambda^{2}$. The limit surface of a generalized tensor-product subdivison algorithm with bi-degree

$$
\operatorname{deg}\left(\mathbf{x}_{0}\right)=d \quad \text { and } \quad \operatorname{deg}\left(\Delta_{12}\right)=2 d-1
$$

is curvature continuous at an extraordinary point $P$ if and only if $P$ is a flat point.

In other words, a generalized box-spline subdivision algorithm can only have a curvature continuous limit surface for $\mu=\lambda^{2}$, if the true degree of the Jacobian $\Delta_{12}$ is less than its formal degree.This is the case, if either one or both of the following conditions hold:

(i) The true degree of $\mathbf{e}^{1}$ or $\mathbf{e}^{2}$ is less than $d$.

(ii) The leading terms in the Jacobian $\Delta_{12}$ cancel.

Since we assume symmetric masks,

$$
\operatorname{deg}\left(\mathbf{e}^{1}\right)=\operatorname{deg}\left(\mathbf{e}^{2}\right)=: d^{\prime} .
$$

If the subdivision surface is curvature continuous and not flat in the extraordinary point and if condition (ii) does not apply then $d^{\prime}<d$ must hold by condition (i). In fact, we compute

- for the total degree $\operatorname{deg}\left(G_{i j}\right)=2\left(2 d^{\prime}+d-4\right)$ and $\operatorname{deg}\left(\Delta_{12}^{4}\right)=4\left(2 d^{\prime}-2\right)$ and

- for the bi-degree $\operatorname{deg}\left(G_{i j}\right)=2\left(2 d^{\prime}+d-2\right)$ and $\operatorname{deg}\left(\Delta_{12}^{4}\right)=4\left(2 d^{\prime}-1\right)$.

Comparing degrees we find in either case that $2 d^{\prime}=d$ and arrive at the following observation:

Observation 6 If the leading terms in the Jacobian $\Delta_{12}$ do not cancel then the limit surface of a generalized box-spline subdivision algorithm is curvature continuous and not flat in an extraordinary point only if the true (bi-)degree of the surface is at least twice the true (bi-)degree of the subdominant eigenfunctions $\mathbf{e}^{1}$ and $\mathbf{e}^{2}$.

This is consistent with the degree estimate of [Reif '96, Zorin '98]. The central idea of these proofs appears already as a parting sentence in [Sabin '91]: the surface is viewed as a function over the tangent plane parametrized by $\mathbf{e}^{1}$ and $\mathbf{e}^{2}$. To have non-zero curvature, it is necessary that the non-tangential component of the surface is at least quadratic in $\mathbf{e}^{1}$ and $\mathbf{e}^{2}$, i.e. $d \geq 2 d^{\prime}$. More generally, if the non-tangential component of the surface is at least of degree $r$ in $\mathbf{e}^{1}$ and $\mathbf{e}^{2}$ then the surface representation has to be at least of degree $r d^{\prime}$. Since $\mathbf{e}^{1}$ and $\mathbf{e}^{2}$ have to have a minimal degree to form $C^{k}$ rings, e.g. $d^{\prime} \geq k+1$ in the tensor-product case, a lower bound, say $r(k+1)$, is deduced [Prautzsch \& Reif '99].

If, on the other hand, the subdivision surface is curvature continuous and not flat in the extraordinary point and if condition (i) does not apply then the leading terms of $\Delta_{12}$ must cancel by condition (ii). Now we compute

- for the total degree $\operatorname{deg}\left(G_{i j}\right)=2 \max \left\{\operatorname{deg}\left(\Delta_{12}\right)+d-2,2(d-1)+d-2\right\}=6 d-8$ and 
- for the bi-degree $\operatorname{deg}\left(G_{i j}\right)=2 \max \left\{\operatorname{deg}\left(\Delta_{12}\right)+d-1,2 d-1+d-1\right\}=6 d-4$.

Comparing against $\operatorname{deg}\left(\Delta_{12}^{4}\right)=4 \operatorname{deg}\left(\Delta_{12}\right)$ we obtain a counterpart to Observation 6.

Observation 7 If the true degree of $\mathbf{e}^{1}$ and $\mathbf{e}^{2}$ is not less than $d$ then the limit surface of a generalized box-spline subdivision algorithm is curvature continuous and not flat in an extraordinary point only if the total degree $\operatorname{deg}\left(\Delta_{12}\right) \leq 3 d / 2-2$, respectively, the bi-degree $\operatorname{deg}\left(\Delta_{12}\right) \leq 3 d / 2-1$.

From these observations, it is evident that the key to curvature continuous subdivision surfaces is the answer to the following question.

Central Question For what choices of eigenfunctions $\mathbf{e}^{1}$ and $\mathbf{e}^{2}$ is $\operatorname{deg}\left(\Delta_{12}\right)$ less than $2 \operatorname{deg}\left(\mathbf{x}_{0}\right)-2$ for total degree, respectively, $2 \operatorname{deg}\left(\mathbf{x}_{0}\right)-1$ for bi-degree generalized box-spline subdivision algorithms?

\section{Curvature continuous subdivision constructions}

If we interpret curvature smoothness in the weak sense of $L^{2}$ integrability, then Observation 4 guarantees that almost all $C^{1}$ subdivision algorithms qualify as curvature smooth. If we allow flat spots, then [Prautzsch \& Umlauf '98a, Prautzsch \& Umlauf '98b] yield low degree, small mask, curvature continuous subdivision algorithms. If we want non-zero bounded curvature, we can adapt the leading eigenvalues as in [Sabin '91, Holt '96]. However, if we want curvature continuity without flat spots the stringent constraints of Lemma 2 apply and a degree-reduced Jacobian in the sense of Observations 6 or 7 is necessary. A trivial example that satisfies these constraints is the regular case of any $C^{2}$ box-spline: here $\mathbf{e}^{1}$ and $\mathbf{e}^{2}$ are linear. The only non-trivial constructions reported to date subdivide a polynomial filling of an $n$-sided hole obtained by projection [Prautzsch '97, Reif '98b].

To see in our newly acquired framework why these constructions yield curvature continuous subdivision surfaces without forced flat points we restate the sufficient conditions derived by Prautzsch [Prautzsch '98]. (Parametrizing the limit surface over the characteristic map, [Reif '98a] concludes also the necessity of these conditions.)

The sufficient conditions of [Prautzsch '98] state, that in order to be able to solve the differential equations (4) it suffices that the eigenfunctions $\mathbf{e}^{3}, \mathbf{e}^{4}$ and $\mathbf{e}^{5}$ be quadratic polynomials in $\mathbf{e}^{1}$ and $\mathbf{e}^{2}$ :

$$
\mathbf{e}^{i}=a_{i}\left(\mathbf{e}^{1}\right)^{2}+b_{i} \mathbf{e}^{1} \mathbf{e}^{2}+c_{i}\left(\mathbf{e}^{2}\right)^{2}, \quad a_{i}, b_{i}, c_{i} \in \mathbb{R}, \quad \text { for } i=3,4,5 .
$$

Indeed the derivatives of $\mathbf{e}^{i}, i=3,4,5$, are then of the form

$$
\begin{aligned}
\mathbf{e}_{u}^{i} & =2 a^{i} \mathbf{e}^{1} \mathbf{e}_{u}^{1}+b^{i}\left(\mathbf{e}_{u}^{1} \mathbf{e}^{2}+\mathbf{e}^{1} \mathbf{e}_{u}^{2}\right)+2 c^{i} \mathbf{e}^{2} \mathbf{e}_{u}^{2}, \\
\mathbf{e}_{u u}^{i} & =2 a^{i}\left(\left(\mathbf{e}_{u}^{1}\right)^{2}+\mathbf{e}^{1} \mathbf{e}_{u u}^{1}\right)+b^{i}\left(\mathbf{e}_{u u}^{1} \mathbf{e}^{2}+2 \mathbf{e}_{u}^{1} \mathbf{e}_{u}^{2}+\mathbf{e}^{1} \mathbf{e}_{u u}^{2}\right)+2 c^{i}\left(\left(\mathbf{e}_{u}^{2}\right)^{2}+\mathbf{e}^{2} \mathbf{e}_{u u}^{2}\right)
\end{aligned}
$$

and analogously for $\mathbf{e}_{v}^{i}, \mathbf{e}_{v v}^{i}$ and $\mathbf{e}_{u v}^{i}$. This yields

$$
\begin{aligned}
\Delta_{1 i} & =\Delta_{12}\left(b^{i} \mathbf{e}^{1}+2 c^{i} \mathbf{e}^{2}\right), \\
\Delta_{2 i} & =-\Delta_{12}\left(2 a^{i} \mathbf{e}^{1}+b^{i} \mathbf{e}^{2}\right) \quad \text { and } \\
D_{u u}^{i} & =2 \Delta_{12}\left(a_{i}\left(\mathbf{e}_{u}^{1}\right)^{2}+b_{i} \mathbf{e}_{u}^{1} \mathbf{e}_{u}^{2}+c_{i}\left(\mathbf{e}_{u}^{2}\right)^{2}\right) .
\end{aligned}
$$

Substituting $D_{u u}^{i}, D_{v v}^{i}$ and $D_{u v}^{i}$ in (3) and (6), the limit terms for the Gauss and the mean curvatures simplify to

$$
K=\sum_{i, j=3,4,5} \frac{P_{i j}}{\left\|\mathbf{p}_{1} \times \mathbf{p}_{2}\right\|^{4}} \cdot f_{i j} \quad \text { and } \quad H=\sum_{\substack{i=3,4,5 \\ k, l=1,2, k \geq l}} \frac{\tilde{P}_{i k l}}{\left\|\mathbf{p}_{1} \times \mathbf{p}_{2}\right\|^{3}} \cdot \tilde{f}_{k l}
$$


where

$$
f_{i j}=\left\{\begin{array}{ll}
4\left(a_{i} c_{j}+a_{j} c_{i}\right)-2 b_{i} b_{j} & \text { for } i \neq j \\
4 a_{i} c_{i}-\left(b_{i}\right)^{2} & \text { for } i=j
\end{array}, \quad \tilde{f}_{k l}=\left\{\begin{array}{ll}
c_{i} & \text { for } k=l=1 \\
a_{i} & \text { for } k=l=2 \\
-b_{i} / 2 & \text { for } k \neq l
\end{array} .\right.\right.
$$

Since these last expressions for the Gauss and mean curvature are constant, the limit surface is curvature continuous at the extraordinary point.

Subdivision algorithms that satisfy the sufficient conditions (8) were derived by [Prautzsch '97, Reif '98b]. We analyze Prautzsch's approach in detail in terms of our necessary and sufficient conditions of Lemma 2. Here $\mathbf{v}_{1}$ and $\mathbf{v}_{2}$ are the eigenvectors to the subdominant eigenvalue $\lambda$ of the Catmull-Clark algorithm. Then $\mathbf{e}^{1}$ and $\mathbf{e}^{2}$ have true bi-degree 3 . Now set $\mathbf{e}^{3}=\left(\mathbf{e}^{1}\right)^{2}, \mathbf{e}^{4}=\mathbf{e}^{1} \mathbf{e}^{2}$ and $\mathbf{e}^{5}=\left(\mathbf{e}^{2}\right)^{2}$ with control nets $\mathbf{w}_{i}, i=3,4,5$. Furthermore, $\mathbf{w}_{1}$ and $\mathbf{w}_{2}$ are the control nets of $\mathbf{e}^{1}$ and $\mathbf{e}^{2}$, respectively, in a degree-doubled representation. Then the subdivision matrix of Prautzsch's construction for is given by

$$
A=M D M^{+}
$$

where

$$
M:=\left[\mathbf{1}, \mathbf{w}_{1}, \mathbf{w}_{2}, \mathbf{w}_{3}, \mathbf{w}_{4}, \mathbf{w}_{5}\right], \quad D:=\operatorname{diag}\left(1, \lambda, \lambda, \lambda^{2}, \lambda^{2}, \lambda^{2}\right), \quad M^{+}:=\left(M^{t} M\right)^{-1} M^{t} .
$$

In this construction the only non-zero eigenvalues of $A$ are $1, \lambda\left(2\right.$-fold), $\lambda^{2}$ (3-fold) corresponding to the eigenvectors $\mathbf{1}, \mathbf{w}_{1}, \ldots, \mathbf{w}_{5}$.

\section{Conclusion}

We surveyed and restated a number of important recent results concerning the curvature continuity of the limit surfaces of generalized box-spline subdivision algorithms. The direct computation of $K$ and $H$ simplifies the matter and yields new insights into why the limit surfaces of most subdivision algorithms are not curvature continuous and what criteria need to be enforced by new constructions.

\section{References}

M.P. Do CARmo (1976). Differential geometry of curves and surfaces. Prentice-Hall Inc., Englewood Cliffs, N.J.

E. CATmull And J. Clark (1978). Recursive generated B-spline surfaces on arbitrary topological meshes. Computer-Aided Design, 10(6):350-355.

F. HoLT (1996). Towards a curvature-continuous stationary subdivision algorithm. Z. Angew. Math. Mech., 76(Suppl. 1):423-424.

C.T. Loop (1987). Smooth Subdivision Surfaces Based on Triangles. Master's thesis, Department of Mathematics, University of Utah, August 1987.

J. Peters And U. ReIF (1998). Analysis of algorithms generalizing B-spline subdivision. SIAM J. Numer. Anal., 35(2):728-748.

H. Prautzsch (1997). Freeform splines. Comput. Aided Geom. Design, 14(3):201-206.

H. PRAutzSCH (1998). Smoothness of subdivision surfces at extraordinary points. Advances in Comp. Math., 9:377-389. 
H. PrautZsCh AND U. ReIF (1999). Degree estimates for $C^{k}$-piecewise polynomial subdivision surfaces. Advances in Comp. Math., 10(2):209-217.

H. Prautzsch and G. Umlauf (1998a). A $G^{2}$-subdivision algorithm. In G. Farin, H. Bieri, G. Brunnett, And T. DeRose, editors (1998a), Geometric Modelling, pages 217-224. Dagstuhl 1996, Computing Supplement 13, Springer-Verlag, 1998.

H. Prautzsch and G. Umlauf (1998b). Improved triangular subdivision schemes. In F.-E. Wolter AND N.M. PATRIKALAKIS, editors (1998b), Computer Graphics International 1998, pages 626632, Hannover, 22.-26. June 1998. IEEE Computer Society.

R. QU (1990). Recursive subdivision algorithms for curve and surface design. PhD thesis, Department of Mathematics and Statistics, Burnel University, Uxbridge, Middlesex, England, August 1990.

U. REIF (1993). Neue Aspekte in der Theorie der Freiformflächen beliebiger Topologie. PhD thesis, Mathematisches Institut A, Universität Stuttgart, Stuttgart, August 1993.

U. REIF (1995). A unified approach to subdivision algorithms near extraordinary vertices. Comput. Aided Geom. Design, 12:153-174.

U. ReIf (1996). A degree estimate for subdivision surfaces of higher regularity. Proc. Amer. Math. Soc., 124(7):2167-2174.

U. REIF (1998a). Analyse und Konstruktion von Subdivisionsalgorithmen für Freiformflächen beliebiger Topologie. Habilitationsschrift, Mathematisches Institut A, Universität Stuttgart, Stuttgart, December 1998. Verlag Shaker, Aachen.

U. REIF (1998b). TURBS - topologically unrestricted rational B-splines. Constr. Approx., 14(1):57-78.

U. REIF AND P. SCHRÖDER (2000). Curvature smoothness of subdivision surfaces. Technical report TR-00-03, Caltech, Pasadena, 2000.

M.A. SABin (1991). Cubic recursive division with bounded curvature. In P.J. Laurent, A. Le MéHauté, And L.L. Schumaker, editors (1991), Curves and Surfaces, pages 411-414. Academic Press, Boston, 1991.

D. Zorin (1998). Stationary Subdivision and Multiresolution Surface Representations. PhD thesis, California Institut of Technology, Pasadena, 1998. 